\title{
$q$-PROBABILITY DISTRIBUTIONS VIA AN EXTENSION OF THE BERNOULLI PROCESS
}

\author{
PHILIP FEINSILVER
}

\begin{abstract}
One-parameter extensions of the Binomial, Negative Binomial, Poisson. Geometric and Gamma distributions are derived via an extension of the standard Bernoulli counting scheme. The method is to examine the elementary "potential theory" for the basic process. Some interesting extensions to continuous time are mentioned also.
\end{abstract}

1. The basic process. We will consider a counting process on the nonnegative integers such that as time goes on the probability of increasing decreases. The related probability distributions are studied using "basic" or "Eulerian" mathematics [8]. We will explain the " $q$ " theory needed as it arises.

Throughout, $x$ is a fixed number, $0<x<1$, corresponding to the probability of success in ordinary Bernoulli trials. $q$ is a fixed parameter, $0<q<1$. The variable $v$ is a complex variable in the domain $(1)=\{|v|<1\}$. We let $S_{n}$ be the location or "number of successes" after $n$ trials. The increments are determined as follows:

At time $n \geqslant 0$ if you are at position $k \geqslant 0$, then, for $S_{0}$ such that $q^{S_{0}} \geqslant x$, we set

$$
\begin{aligned}
& P\left(S_{n+1}=k \mid S_{n}=k\right)=1-q^{n-k} x, \\
& P\left(S_{n+1}=k+1 \mid S_{n}=k\right)=q^{n-k} x .
\end{aligned}
$$

The process $S_{n}$ is a discrete parameter Markov process, although it is neither temporally nor spatially homogeneous.

For a bounded function $f(k)$, set $\operatorname{Pf}(n, a)=\left\langle f\left(S_{n}\right)\right\rangle_{a},\langle\rangle_{a}$ denoting expected value with respect to the measure on paths starting at $a$. We derive from (1.1) and (1.2),

$$
\begin{aligned}
& P f(n+1, a)-P f(n, a)=\left\langle q^{n-S_{n} x}\left[f\left(S_{n}+1\right)-f\left(S_{n}\right)\right]\right\rangle_{a}, \\
& P f(0, a)=f(a) .
\end{aligned}
$$

In this context the useful functions are of the form $f_{v}(k)=\prod_{j=0}^{k-1}\left(1-v q^{j}\right) \equiv(v)_{k}$, using the abbreviated standard notation, for $0<k \leqslant \infty$. Also set $(v)_{0} \equiv 1,(v)_{k}=0$ for $k<0$. Then for $k \geqslant 0$,

$$
\begin{gathered}
(v)_{k+1}-(v)_{k}=-q^{k} v(v)_{k} \quad \text { yields } \\
P f_{v}(n+1, a)=\left(1-x v q^{n}\right) P f_{v}(n, a), \quad P f_{v}(0, a)=(v)_{a} .
\end{gathered}
$$

Thus, $P f_{v}(n, a)=(v)_{a}(x v)_{n}$.

Received by the editors February 27, 1981 and, in revised form, June 25, 1982.

1980 Mathematics Subject Classification. Primary 60J75; Secondary 60J15.

त) 1983 American Mathematical Society $0002-9939 / 82 / 0000-0721 / \$ 02.50$ 
For the process starting at 0 (we drop the subscript 0 ) this yields

$$
\left\langle(v)_{S_{n}}\right\rangle=(x v)_{n} .
$$

It is useful to consider briefly generating functions of the form $\phi(v)=\sum_{k=0}^{\infty}(v)_{k} p_{k}$ for probability distributions $p_{k}$. We denote an "Eulerian derivative" by

$$
W f(v)=[f(v)-f(Q v)] / v Q
$$

where $Q=q^{-1}$. Note that $(q)_{n}=\prod_{j=1}^{n}\left(1-q^{j}\right)$. Then we have

Proposition 1.1. Map the distribution $\left\{p_{n}\right\} \rightarrow \phi(v)=\Sigma_{0}^{\infty} p_{k}(v)_{k}$. Then:

(a) $\phi$ is holomorphic on $\mathscr{D}$,

(b) $p_{n}=\left.(q)_{n}^{-1} W^{n} \phi(v)\right|_{v=1}$ is the inverse transform.

Proof. (a) Observe the estimate

$$
\sup _{K, n}\left|\frac{\partial}{\partial v}(v)_{n}\right| \leqslant \frac{1}{1-q} \sup _{K} \frac{1}{1-|v|} \exp \left(\frac{1}{1-q}\right)
$$

for any compact subset $K$ of $\mathscr{Q}$.

(b) Inductively, check that $W^{m}(v)_{k}=(q)_{k}(v)_{k-m} /(q)_{k-m}$.

Applying Proposition 1.1 to (1.5) yields the $q$-binomial distribution

$$
P\left(S_{n}=k\right)=\left(\begin{array}{l}
n \\
k
\end{array}\right)_{q} x^{k}(x)_{n-k},
$$

where $\left(\begin{array}{l}n \\ k\end{array}\right)_{q}$ denotes the $q$-binomial coefficient

$$
\left(\begin{array}{l}
n \\
k
\end{array}\right)_{q}=\frac{(q)_{n}}{(q)_{k}(q)_{n-k}}
$$

The identity $P\left(S_{n}<\infty\right)=1$ yields a $q$-binomial theorem

$$
\sum_{k=0}^{n}\left(\begin{array}{l}
n \\
k
\end{array}\right)_{q} x^{k}(x)_{n-k}=1
$$

The usual conventions $\left(\begin{array}{l}n \\ k\end{array}\right)_{q}=1, k=0,0$ for $k<0$ will be assumed.

The generating function extension of (1.9) is the formulation usually called "the" $q$-binomial theorem [2],

$$
\sum_{0}^{\infty} \frac{v^{n}(x)_{n}}{(q)_{n}}=\frac{(x v)_{\infty}}{(v)_{\infty}}
$$

This follows by applying $\sum_{0}^{\infty} v^{n} /(q)_{n}$ to both sides of (1.9) and then using Euler's identity

$$
(x)_{\infty}^{-1}=\sum_{0}^{\infty} \frac{x^{n}}{(q)_{n}} .
$$

Using (1.5) we can derive (1.10) directly and thus provide a probabilistic proof of Euler's identity: Combine (1.5) and (1.7) as

$$
\begin{aligned}
\left\langle(v)_{S_{n}}\right\rangle & =\sum_{0}^{\infty}\left(\begin{array}{l}
n \\
k
\end{array}\right)_{q} x^{k}(x)_{n-k}(v)_{k}=(x v)_{n} \\
& =\sum_{0}^{\infty} \frac{(q)_{n}}{(q)_{n-k}(q)_{k}} x^{k}(x)_{\infty}(v)_{k}\left(x q^{n-k}\right)_{\infty}^{-1}
\end{aligned}
$$


As $n \rightarrow \infty,(q)_{n-k} /(q)_{n} \rightarrow 1(k$ fixed $), q^{n} \rightarrow 0$. Thus,

$$
\lim _{n \rightarrow \infty}\left\langle(v)_{s_{n}}\right\rangle=\sum_{0}^{\infty} \frac{x^{k}(v)_{k}}{(q)_{k}} \cdot(x)_{\infty}=(x v)_{\infty} .
$$

II. Waiting time distributions. Distribution of the maximum. To get at the "structure" of the process $S_{n}$ we consider the waiting times $T_{m}=\min \left\{n: S_{n}=m\right\}$. By the Markov property

$$
\begin{aligned}
& P\left(T_{m}=n\right)=P\left(S_{n-1}=m-1\right) \cdot P\left(S_{n}=m\right) \\
&=\left(\begin{array}{c}
n-1 \\
m-1
\end{array}\right)_{q} x^{m-1}(x)_{n-m} \cdot q^{n-m} x \\
&=\left(\begin{array}{c}
n-1 \\
m-1
\end{array}\right)_{q} x^{m} q^{n-m}(x)_{n-m} \text { or }\left(\begin{array}{c}
m+k-1 \\
k
\end{array}\right)_{q} x^{m} q^{k}(x)_{k}, \\
& k=n-m .
\end{aligned}
$$

This is the $q$-version of the negative binomial distribution. In particular,

$$
P\left(T_{1}=n\right)=x q^{n-1}(x)_{n-1},
$$

a q-geometric distribution.

Introduce the functions $f_{m}(x)=P\left(T_{m}<\infty\right)$, i.e.

$$
f_{m}(x)=x^{m} \sum_{k \geqslant 0}\left(\begin{array}{c}
m+k-1 \\
k
\end{array}\right)_{q} q^{k}(x)_{k} .
$$

We can show that the process eventually "sticks" with probability one.

PROPOSITION 2.1. (a) $\sup _{m>0} f_{m}(x)=f_{1}(x)<1$.

(b) $P\left(T_{m}<\infty\right.$ for only finitely many $\left.m\right)=1$.

Proof. (a) Note that $P\left(T_{m+1}<\infty\right) \leqslant P\left(T_{m}<\infty\right)$. From $f_{1}(x)=\Sigma_{k} x q^{k}(x)_{k}$,

$$
f_{1}(q x)=\left(f_{1}(x)-x\right) /(1-x)
$$

since $(q x)_{k}=(x)_{k+1} /(1-x)$.

Suppose for some $a \in(0,1), f_{1}(a)=1$. Then by $(2.4), \lim _{n \rightarrow \infty} f_{1}\left(q^{n} a\right)=1$. But $f_{1}(x)$ extends continuously to $[0,1]$ since $\sup _{0<x<1} f_{1}(x) \leqslant 1 /(1-q)$. Thus $\lim _{n \rightarrow \infty} f_{1}\left(q^{n} a\right)=0$. Hence $f_{1}(x)<1$ for all $0<x<1$.

(b) $f_{m}(x)$ can be written $\Sigma_{k}\left(q^{k+1}\right)_{m-1} x^{m}(q)_{m-1}^{-1} q^{k}(x)_{k}$ for $m \geqslant 1$. Sum from 1 to $\infty$ and apply (1.10):

$$
\begin{aligned}
\sum_{m=1}^{\infty} f_{m}(x) & =x \sum_{k}\left(q^{k+1} x\right)_{\infty} q^{k}(x)_{k} /(x)_{\infty} \\
& =x \sum_{k} q^{k}(x)_{k} /(x)_{k+1}=x \sum_{k} q^{k} /\left(1-x q^{k}\right)<\infty
\end{aligned}
$$

The Borel-Cantelli Lemma applies to yield the result.

This very interesting feature of the process is that it is bounded with probability one.

A recursion formula for the $f_{m}$ provides further insight into Proposition 2.1(a).

Introduce the operators:

$z: z f(v)=f(q v)$ 
$y: y f(v)=v f(v)$ and

$w=(1-y) z$ (operator composition understood).

It is easy to see that

$$
w y=q y w \text { and } w^{k}=(y)_{k} z^{h} .
$$

The important relations are

$$
w^{m} y^{n}=q^{m n} y^{n} w^{m} \quad \text { and } \quad x^{k} 1=(x)_{k} .
$$

where 1 denotes the constant function identically one.

Proposition 2.2. (a) $f_{m}(x)=x^{m}(q w)_{m}^{-1} 1$.

(b) $f_{m+1}(x)=x \sum_{k} q^{k}(x)_{k} f_{m}\left(q^{k} x\right)$.

Proof.

(a)

$$
\begin{aligned}
f_{m}(x) & =x^{m} \sum_{k}\left(q^{m}\right)_{k} q^{k}(x)_{k} /(q)_{k} \\
& =x^{m} \sum_{k}\left(q^{m}\right)_{k}(q w)^{k} 1 /(q)_{k} \\
& =x^{m}\left(q^{m+1} w\right)_{\infty} /(q w)_{\infty} 1 \quad(\text { by }(1.10)) \\
& =x^{m}(q w)_{m}^{-1} 1 . \\
f_{m+1}(x) & =x^{m+1}(w q)_{m}^{-1}\left(1-w q q^{m}\right)^{-1} 1 \\
& =x^{m+1} \sum_{k}(w q)^{k} q^{k m}(w q)_{m}^{-1} 1 \\
& =x \sum_{k} q^{k} w^{k} y^{m}(w q)_{m}^{-1} 1 \quad \text { by }(2.7) \\
& =x \sum_{k} q^{k}(x)_{k} f_{m}\left(q^{k} x\right) \quad \text { by }(2.6) \text { and }(2.7) .
\end{aligned}
$$

(b)

From (b) it readily follows inductively that $f_{m+1}(x) \leqslant f_{1}(x)<1$.

We next derive the distribution of $M=\max S_{n}=\lim _{n \rightarrow \infty} S_{n}$ and consequently, explicitly evaluate $f_{m}(x)$.

Since $P\left(\max S_{n}<\infty\right)=1$, by Proposition 2.1, we can let $n \rightarrow \infty$ in (1.5) to get

$$
\left\langle(v)_{M}\right\rangle=(x v)_{\infty}
$$

Now Proposition 1.1 yields, using $W(x v)_{\infty}=x(x v)_{\infty}$

$$
P(M=k)=(x)_{\infty} x^{k} /(q)_{k},
$$

\section{a $q$-Poisson distribution.}

Since

$$
P(M=m)=P\left(T_{m}<\infty\right)-P\left(T_{m+1}<\infty\right)
$$

or

$$
(x)_{\infty} x^{m} /(q)_{m}=f_{m}(x)-f_{m+1}(x)
$$

it follows, with $f_{0}(x)=1$, that

$$
f_{m}(x)=1-(x)_{\infty} \sum_{j=0}^{m-1} x^{j} /(q)_{j}
$$


In particular,

$$
f_{1}(x)=1-(x)_{\infty} .
$$

Next we consider some extensions.

III. Limiting distributions. Extensions to continuous time. As for the usual Poisson limit theorem we set $N \geqslant 1, x \rightarrow \lambda / N, q \rightarrow q^{1 / N},[N t]=T$. Denote by $X(t)$ the limiting process: $S_{n} \rightarrow S_{T} \rightarrow X(t)$ as $N \rightarrow \infty$. The distribution for $S_{n}$ yields

$$
\begin{aligned}
P(X(t)=k) & =\lim _{N \rightarrow \infty}\left(\begin{array}{l}
T \\
k
\end{array}\right)_{q} x^{k}(x)_{T-k} \\
& =\lim _{N \rightarrow \infty}\left(\begin{array}{l}
T \\
k
\end{array}\right)_{q^{1 / N}}\left(\frac{\lambda}{N}\right)^{k}\left(\frac{\lambda}{N} ; q^{1 / N}\right)_{T-k} \\
& =\left(\lambda l\left(1-q^{t}\right)\right)^{k} \exp \left(-\lambda l\left(1-q^{t}\right)\right) / k ! .
\end{aligned}
$$

The key limit relation is $\lim _{N \rightarrow \infty} N\left(1-q^{k / N}\right)=k / l$, where $l \log Q=1$.

The transition probabilities are as follows:

$$
P(\text { jump in an interval } t, t+d t)=\lim _{N \rightarrow \infty} q^{\left(T-s_{T}\right) / N} \lambda N^{-1}=q^{t} \lambda d t .
$$

Thus we have a Poisson process where the intensity is exponentially damped. This is a standard type of jump Markov process (see e.g. [5]).

The waiting time distributions yield, taking $N \rightarrow \infty$,

$$
P\left(T_{m} \sim(t, t+d t)\right)=\left(1-q^{t}\right)^{m-1}(\lambda l)^{m} q^{t} \exp \left(-\lambda l\left(1-q^{t}\right)\right) d t / l \Gamma(m) .
$$

The total mass

$$
P\left(T_{m}<\infty\right)=1-e^{-\lambda l} \sum_{j=0}^{m-1} \frac{(\lambda l)^{j}}{j !} .
$$

The basic expectation $\left\langle(v)_{S_{n}}\right\rangle=(x v)_{n}$ becomes

$$
\left\langle(1-v)^{X(t)}\right\rangle=\exp \left(-v \lambda l\left(1-q^{t}\right)\right)
$$

This process is readily understood by standard methods. The new feature to come is that the $q$-Bernoulli measures have direct extensions to continuous-parameter distributions; a situation unlike that for the standard case $q=1$.

Consider a process with $q \rightarrow q^{N}:\left\langle\left(v ; q^{N}\right)_{S_{T}}\right\rangle=\left(x v ; q^{N}\right)_{T}$. The jump probability is $x q^{N\left(n-S_{n}\right)}$ at position $\left(n, S_{n}\right)$. If we leave $S_{n}$ the same, denoting it by $S_{n}^{N}$, and rescale $q \rightarrow q^{1 / N}$, the jump probability is $x q^{n-S_{n}^{N}}$. This heuristically gives us a motivation for considering distributions satisfying

$$
\left\langle(v)_{k(t)}\right\rangle=(x v)_{t} .
$$

$$
\text { (For } t \geqslant 0,(v)_{t} \text { is defined by }(v)_{\infty} /\left(v q^{t}\right)_{\infty} \text {.) }
$$

First we ask: What is the distribution of $S_{n}^{N}$ ? For large $N$, it turns out to be approximately geometric. The following is easily checked: 
Proposition 3.1. (a) As $q \rightarrow 0$, the distribution of $S_{n}$ tends to a finite geometric distribution $g_{n}$ :

$$
\begin{gathered}
P\left(g_{n}=k\right)=x^{k}(1-x), \quad 0 \leqslant x<n, \\
P\left(g_{n}=n\right)=x^{n} .
\end{gathered}
$$

(b) As $q \rightarrow 0$, the distribution of the maximum $M$ tends to a geometric distribution.

Briefly, as $q \rightarrow 1$ we have Bernoulli and Poisson distributions, as $q \rightarrow 0$ we have geometric distributions. Next we consider directly the extension suggested in (3.6), going from $n$ discrete to $t$ continuous. We summarize the results to be discussed:

THEOREM 3.2. (1) For any $q$ and fixed $x=q^{\delta}, 0<\delta<1$, the distribution of $k(t)$ satisfying (3.6) is a probability distribution for all $t$ satisfying $1-\delta<\operatorname{fract}(t) \leqslant 1$, where fract denotes the fractional part of $t$.

(2) For any $q$ and fixed $x=q^{\delta}, 1<\delta<2$, relation (3.6) determines a probability distribution for all $t$ satisfying $0 \leqslant \operatorname{fract}(t)<2-\delta$.

(3) For any $q$ and $x=q$ the distribution of $k(t)$ is positive and has total mass $(q)_{t} /(q)_{\infty}$ for $t$ nonintegral. For integer values of $t$, we have the usual $q$-Bernoulli distribution.

(4) In all cases as $t \rightarrow \infty$, the limiting distribution is the q-Poisson (2.9).

First calculate using Proposition 1.1 what the probabilities should be:

$$
p_{k}=\left.(q)_{k}^{-1} W^{k}(x v)_{t}\right|_{v=1}=\frac{(q)_{t}}{(q)_{k}(q)_{t-k}} x^{k}(x)_{t-k} \quad \text { (see 3.6a). }
$$

This can be written alternatively as

$$
p_{k}=\frac{(x)_{\infty}}{(x \tau)_{\infty}} \frac{\left(q \tau Q^{k}\right)_{k}}{\left(x \tau Q^{k}\right)_{k}} \frac{x^{k}}{(q)_{k}}
$$

where $\tau=q^{t}$.

The expression in (3.8) shows that for the $p_{k}$ to be positive there must be an integer $j \geqslant 0$ such that $q^{j}>q \tau>q^{j+1}$ and $q^{j}>x \tau>q^{j+1}$ simultaneously. Consideration of the cases where $x=q^{\delta}, 0<\delta<1$ or $1<\delta<2$ yields 1 and 2 of Theorem 3.2. For $x=q$,

$$
p_{k}=(q)_{t} q^{k} /(q)_{k} \text { with } \sum_{k=0}^{\infty} p_{k}=(q)_{t} /(q)_{\infty}=(q \tau)_{\infty}^{-1} .
$$

Finally it remains to check that for $x \neq q, \sum p_{k}=1$.

Recall the well-known relation (see e.g. [1])

$$
(x)_{m}=\sum_{k}\left(\begin{array}{c}
m \\
k
\end{array}\right)_{q}(-1)^{k} q^{\left(\frac{k}{2}\right)} x^{k}
$$

which may be checked by applying $W^{k}$ at $x=0$. Letting $m \rightarrow \infty$ yields the Eulerian identity

$$
(x)_{\infty}=\sum_{k} \frac{x^{k}(-1)^{k} q^{\left(\frac{k}{2}\right)}}{(q)_{k}} .
$$


The calculation proceeds as follows:

$$
\sum_{k} p_{k}=\frac{(x)_{\infty}}{(x \tau)_{\infty}} \sum_{k} \frac{x^{k}}{(q)_{k}} \frac{(x \tau)_{\infty}}{(q \tau)_{\infty}} \frac{\left(q \tau Q^{k}\right)_{\infty}}{\left(x \tau Q^{k}\right)_{\infty}}
$$

from (3.8).

Apply (1.10) to write this last fraction as

$$
\sum_{j} \frac{(x \tau)^{j} Q^{k j}\left(q x^{-1}\right)_{j}}{(q)_{j}}
$$

Summing over $k$ and writing $x^{j}\left(q x^{-1}\right)_{j}=q^{\left(\frac{1}{2}\right)}(-q)^{j}\left(x Q^{j}\right)_{j}$ yields

$$
\frac{(x)_{\infty}}{(q \tau)_{\infty}} \sum_{j} \frac{(q \tau)^{j}(-1)^{j} q^{\left(\frac{1}{2}\right)}}{(q)_{j}} \frac{\left(x Q^{j}\right)_{j}}{\left(x Q^{j}\right)_{\infty}}=\frac{(q \tau)_{\infty}}{(q \tau)_{\infty}}=1
$$

by (3.11). This completes the proof of Theorem 3.2.

IV. $q$-gamma distributions. For given $q$ and $x,(2.3)$ and (2.12) yield the identities

$$
\begin{aligned}
P\left(T_{m}<\infty\right) & =f_{m}(x)=x^{m} \sum_{k=0}^{\infty}\left(\begin{array}{c}
m+k-1 \\
k
\end{array}\right)_{q} q^{k}(x)_{k} \\
& =1-(x)_{\infty} \sum_{j=0}^{m-1} \frac{x^{j}}{(q)_{j}} .
\end{aligned}
$$

Recall from the standard theory of the Poisson Process the relation

$$
e^{-x} \sum_{j=0}^{m-1} \frac{x^{j}}{j !}=\int_{x}^{\infty} s^{m-1} e^{-s} d s / \Gamma(m) .
$$

Let us see the $q$-version of this. Define the measure

$$
d \psi(s)=\sum_{j=0}^{\infty} \delta\left(s-q^{j}\right) q^{j} \quad \text { on } 0 \leqslant s \leqslant 1 .
$$

Set $\int_{0}^{x} f(s) d \psi(s)=\int_{0}^{1} f(s x) x d \psi(s)$ for $x>0$.

THEOREM 4.1.

$$
f_{m}(x)=\int_{0}^{x} s^{m-1}(s q)_{\infty} d \psi(s) /(q)_{m-1}
$$

Proof. Write

$$
f_{m}(x)=\frac{x^{m}}{(q)_{m-1}} \sum_{k}(q)_{k+m-1} q^{k}(x)_{k} /(q)_{k} .
$$

The integral expression equals

$$
\frac{x^{m}}{(q)_{m-1}} \sum_{j} q^{j m}\left(x q q^{j}\right)_{\infty} .
$$


Cancelling $x^{m} /(q)_{m-1}$, continue with (4.3) as follows:

$$
\begin{aligned}
\sum_{k}\left((q)_{\infty} /\left(q^{k+m}\right)_{\infty}\right) q^{k}(x)_{k} /(q)_{k}=\sum_{j} \sum_{k}(q)_{\infty} q^{j k} q^{j m} q^{k}(x)_{k} /(q)_{j}(q)_{k} \\
\quad=\sum_{j} q^{j m}(q)_{\infty}(q)_{j}^{-1}\left(q q^{j} x\right)_{\infty} /\left(q q^{j}\right)_{\infty} \text { by }(1.10) \\
=\sum_{j} q^{j m}\left(q q^{j} x\right)_{\infty}
\end{aligned}
$$

in agreement with (4.4).

REMARKS. Thus the $q$-gamma distribution (4.2) represents the "hitting probability" for our basic process. Note that $f_{m}(1)=1$, so that $x$ has its "natural domain": $0<x<1$.

\section{REFERENCES}

1. (j. E. Andrews, The theorn of partitions, Addison-Wesley, Reading. Mass., 1976.

2. R. Askey. Ramanujan's extensions of the gamma and heta functions, Amer. Math. Monthly 87 (1980), 346-359.

3. J. Cigler, Operatormethoden für q-Identitäten, Monatsh. Math. 88 (1979). 87-105.

4. P. Feinsilver, Commutators, anti-commutators, and Eulerian calculus, Rocky Mountain J. Math. 12 (1982), 171-183.

5. 1. I. Gikhman and A. V. Skorokhod, Introduction to the theory of random processes, Saunders. Philadelphia, Pa.. 1969.

6. W. Hahn, Üher die Höheren Heineschen Reihen und eine einheitliche Theorie der sogenannten speziellen Funktionen, Math. Nachr. 3 (1949/50), 257-294.

7. F. H. Jackson, Basic integration, Quart. J. Math. Oxford Ser. 2 (1951), 1-6.

8. L. J. Slater, Generalized hypergeometric functions, Cambridge Univ. Press, New York, 1966.

Department of Mathematics, Southern Illinois University, Carbondale, Illinois 62901 